\title{
Lapurdum
}

LAPURDUM Euskal ikerketen aldizkaria | Revue d'études basques |

Revista de estudios vascos | Basque studies review

$7 \mid 2002$

Numéro VII

\section{L'antipassif basque et l'hypothèse de Levin}

\section{Rudolf P.G. de Rijk}

URL : http://journals.openedition.org/lapurdum/1027

DOI : 10.4000/lapurdum.1027

ISSN : 1965-0655

Éditeur

IKER

Édition imprimée

Date de publication : 1 octobre 2002

Pagination : 295-312

ISBN : 2-86781-321-2

ISSN : $1273-3830$

\section{Référence électronique}

Rudolf P.G. de Rijk, «L'antipassif basque et l'hypothèse de Levin », Lapurdum [En ligne], 7 | 2002, mis en ligne le 01 juin 2009, consulté le 19 avril 2019. URL : http://journals.openedition.org/lapurdum/1027 ; DOI : 10.4000/lapurdum.1027 


\title{
L'antipassif basque et l'hypothèse de Levin
}

\author{
Rudolf P.G. de RIJK
}

Université de Leiden

IKER UMR 5478 CNRS

\section{Préliminaires}

Cet article comprend deux parties. Le but de la première est d'attirer l'attention des grammairiens sur l'existence d'une diathèse antipassive en basque ancien. La seconde partie, reprenant l'hypothèse de la linguiste américaine Beth $\mathrm{C}$. Levin sur l'inaccusativité des verbes intransitifs en basque, cherche à établir la validité de cette hypothèse pour une étape antérieure de la langue et se termine en signalant les conséquences de cet état de choses pour la typologie du basque à cette époque. Ce qui lie les deux parties, c'est l'idée défendue par l'auteur que la plupart des verbes inergatifs se sont produits par détransitivisation du verbe transitif correspondant. Or, parmi les procédés de détransitivisation qu'a connus le basque au cours de l'histoire, l'antipassif n'est nullement négligeable, encore qu'il ait été négligé jusqu'à présent par la totalité des grammairiens. Précisons d'emblée que l'essai qu'on va lire se situera à peu près entièrement sur le plan observationnel, le soin d'intégrer les phénomènes traités dans une théorie de syntaxe générale étant laissé à des plumes plus compétentes.

\section{Première partie : l'antipassif}

Avant d'entamer l'étude des faits basques, il me semble utile de nous entendre sur le sens précis du terme antipassif. A ce propos, cette espèce de répertoire des idées reçues qu'est le Dictionnaire de linguistique de la librairie Larousse ne nous est d'aucun secours: nulle mention n'y est faite de l'antipassif, bien qu'il y ait une page entière dédiée au passif. Et même le Nouveau dictionnaire encyclopédique des sciences du langage de Ducrot et Schaeffer, manuel admirable sous plusieurs points de vue, ne contient ni le terme ni la notion correspondante. Pour la définition souhaitée, force nous est de recourir à une publication anglo-américaine, en l'occurrence l'International Encyclopedia of Linguistics, rédigée par William Bright et ses collaborateurs. Dans un article de la main de Robert M.W. Dixon intitulé "Australian Languages" on trouve la définition suivante: "... the ANTIPASSIVE derivation: deep A (c.-à-d. le sujet transitif) becomes surface $S$ (c.-àd. le sujet intransitif), deep $O$ (c.-à-d. l'objet direct) is marked by an oblique case (dative, locative, or instrumental, in different languages), and the verb bears an antipassive derivational suffix. » (op. cit. I, 136).

$\mathrm{Au}$ bénéfice du lecteur peu accoutumé au style anglo-saxon il convient de relever qu'il s'agit à proprement parler d'une diathèse, d'une organisation 
alternative de la phrase transitive qui, tout en changeant les marques casuelles, n'altère point les rôles thématiques des syntagmes nominaux.

On observera d'ailleurs que la clausule finale a été gauchement formulée. Comme il s'agit d'une tentative de définition de la dérivation antipassive, il est carrément circulaire d'invoquer un "suffixe dérivationnel antipassif". Du reste, un marqueur quelconque suffit, pas forcément un suffixe comme dans les langues indigènes australiennes étudiées par M. Dixon.

Pour plus de clarté, nous allons comparer de façon schématique le passif à l'antipassif.

PASSIF :

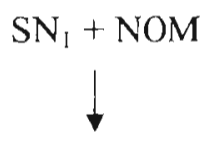

CAS OBLIQUE
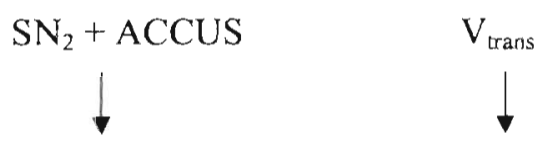

$\mathrm{SN}_{2}+\mathrm{NOM}$

$V_{\text {pass }}$

$\mathrm{SN}_{1}+\mathrm{ERG}$

$\mathrm{SN}_{2}+\mathrm{ABS}$

$\mathrm{V}_{\text {trans }}$

ANTIPASSIF :
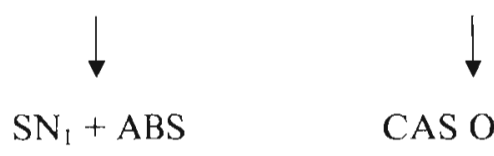

CAS OBLIQUE

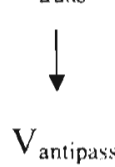

Par l'opération du passif, l'actant le plus élevé (c.-à-d. le sujet de la phrase) se dégrade en syntagme adverbial, après quoi le syntagme nominal qui survit va servir de sujet et perdre sa marque accusative, sauf dans de rares cas, comme en ukrainien, où la marque accusative se maintient. Cette exception mise à part, on constate donc un double changement de cas: le nominatif se fait oblique en même temps que l'accusatif devient nominatif.

Scrutons maintenant le procédé antipassif, qui est censé opérer sur une structure de phrase ergative. Cette fois, c'est l'actant structuralement le plus bas, donc le syntagme absolutif servant d'objet direct, qui se dégradera en syntagme adverbial. De ce fait, la structure cesse d'être transitive, et le syntagme nominal sujet, initialement ergatif, se mue en absolutif. Voilà donc également un double changement de cas: l'absolutif se fait oblique en même temps que l'ergatif devient absolutif.

Maintenant que nous sommes au clair sur ce qu'il faut entendre par la notion d'antipassif, on voudra bien me permettre d'ouvrir une parenthèse à propos de l'histoire de ce vocable. Car, s'il est exact que l'idiome dyirbal décrit si magistralement par M. Dixon fournit un exemple modèle de l'antipassif, ce chercheur n'est ni le découvreur du phénomène comme conception théorique, ni l'auteur du terme devenu courant parmi les syntacticiens. Malheureusement, je me vois obligé d'avouer que j'ignore qui, au juste, a découvert le premier le phénomène en question. En toute probabilité, les savants russes ou soviétiques qui travaillaient sur la langue avare du Caucase ou sur certains idiomes de la vaste Sibérie en avaient déjà pleine conscience bien avant la seconde guerre mondiale. Mais toute information là-dessus me fait défaut. Toujours est-il que, dès 1946, l'antipassif était familier à l'éminent savant polonais Jerzy Kuryłowicz comme 
procédé régulier de détransitivisation dans les langues ergatives. On peut s'en rendre compte en lisant l'article de sa main "Ergativnost i stadial'nost v jazyke", traduit en français trois ans après sous le titre "La construction ergative et le développement stadial du langage". Tout est là, sauf le terme.

Ce terme, nous le devons au linguiste américain Michael Silverstein. C'est lui qui se servit le premier du terme antipassif dans un exposé technique intitulé "Chinook Verb Thematization and Underlying Ergativity" présenté devant le Harvard Linguistic Circle au printemps de l'an 1969. Par la suite, M. Silverstein fit encore, sous le même titre, un exposé contenant également le terme en question, ceci dans le cadre plus officiel de la "8th Conference on American Indian Languages", qui eut lieu à la Nouvelle Orléans le 21 novembre de la même année. Comme cet exposé n'a jamais été publié comme tel -il s'incorpore en quelque sorte dans un article du titre "Hierarchy of Features and Ergativity" paru en 1976il faut attendre l'an 1972 pour que le mot antipassif soit attesté par écrit. C'est toujours à propos du chinook, car il s'agit du mémoire intitulé "Chinook Jargon: Language Contact and the Problem of Multilevel Generative Systems, part I" publié par M. Silverstein dans la revue Language (48-2, 378 -406). A la page 395 de cet article on peut lire ceci: "I have termed this -ki- form the ANTIPASSIVE construction, playing upon its inverse equivalence to a passive of accusative languages, because the sense is clearly equivalent to a transitive, though the form is intransitive, with the grammatical function of the remaining noun phrase reversed (ergator becomes non-ergator). " Assez rapidement, au bout d'une douzaine d'années, le terme s'est imposé effectivement parmi les spécialistes des langues ergatives amérindiennes, comme on peut le constater en feuilletant simplement le volume 16 de la série Syntax and Semantics, qui est dédié à la syntaxe des langues indigènes de l'Amérique.

Ayant fermé la parenthèse, posons enfin la question qui nous intéresse: Le basque, langue à morphologie ergative, possède-t-il, oui ou non, un antipassif? Eh bien, à en croire Jeffrey Heath, il faut répondre: "Oui, mais c'est un antipassif non-syntaxique. ")

Dans un article intitulé "Antipassivization: A Functional Typology" paru en 1976 dans Proceedings of the Second Annual Meeting of the Berkeley Linguistics Society, M. Heath arrive à cette conclusion inattendue en comparant la structure de la forme verbale de présent $d u t$, 'je l'ai', à celle de la forme correspondante au passé: nuen, 'je l'avais'. L'analyse de ces formes se déroule ainsi: la racine du verbe étant $-u$-, le $-t$ final de dut marque la première personne du singulier en fonction ergative. Or, ce $-t$ manque dans la forme du passé nuen, où la première personne du singulier est représentée par un préfixe $n$-, qui, en d'autres formes, marque la fonction absolutive. De plus, le $d$ - initial de $d u t$, allomorphe de $d a$ figurant dans d'autres verbes, est interprété par $M$. Heath comme une marque de fonction absolutive correspondant à l'objet du verbe, de sorte que la disparition de cette marque dans la forme nuen complèterait le tableau et permettrait d'affirmer que le passage du présent au passé se faisait formellement au moyen d'un véritable procédé antipassif.

L'auteur de l'article en reste là. Cependant, si l'on admet que la morphologie d'aujourd'hui reflète la syntaxe d'hier, il est permis de penser qu'un basque plus ancien possédait un antipassif syntaxique s'appliquant aux structures ergatives fondées sur une certaine catégorie de verbes. 
Bien entendu, il faut corriger le raisonnement de l'auteur sur un détail. Comme je l'ai montré dans le sillage de l'érudit basque J. Oregi Aranburu, le préfixe $d(a)$ est une marque de présent et nullement une marque absolutive de troisième personne comme l'enseignait la tradition grammaticale suivie par M. Heath (voir mon essai "Nunc' vasconice", section 7).

On peut soutenir que cette correction n'infirme guère la conclusion obtenue. En effet, le principal c'est que le passé présente un préfixe $n$-absolutif de première personne qui paraît indiquer une structure intransitive, comme dans $n$-a-tor, 'je viens', là où la forme correspondante du présent montre un suffixe $-t$ de valeur ergative.

Faudra-t-il donc se ranger du côté de $\mathrm{M}$. Heath? J'avoue que je reste indécis. Ce qui me gêne, c'est que je n'arrive pas à voir comment le procédé antipassif aurait fini par se faire obligatoire dans un énoncé au passé tout en étant impossible dès qu'il s'agit du présent.

D'autre part, l'hypothèse de cet antipassif permet de résoudre un des mystères de la morphologie basque: la question de savoir pourquoi la caractéristique du passé dans le verbe se manifeste tantôt par - $n$, tantôt par - en. Dans cette hypothèse, l'épenthèse régulière après consonne mise à part, elle aurait la forme invariable $-n$, tandis que l'e post-vocalique qu'on trouve dans des formes comme nuen 'je l'avais', nioen 'je le disais', nekien 'je le savais', ne serait autre que le marqueur de l'antipassif, qui naturellement fait défaut dans des formes comme ninduzun "vous m'aviez', zintudan 'je vous avais', formes qui n'ont pas subi ce procédé.

Laissons donc la question ouverte et continuons l'investigation en précisant que nous sommes à la recherche d'un procédé syntaxique qui mette en rapport une structure ergative avec une structure intransitive, bref, un procédé de détransitivisation, non plus morphologique, mais syntaxique.

Chemin faisant, on ne peut pas ne pas croiser l'oeuvre du spécialiste de la syntaxe basque, M. Georges Rebuschi. En effet, un des premiers travaux de ce chercheur infatigable porte pour titre "Autour du passif et de l'antipassif en basque biscayen" et s'adresse donc directement au thème qui nous occupe. Le texte datant de 1978 fait partie du volume Relations Prédicat-actant(s) dans des langues de types divers II, édité par Mme Catherine Paris en 1979 (SELAF, Paris). Au début de l'article figure la définition suivante: "L'antipassif se définit dans les langues à morphologie ergative comme une paraphrase marquée de la structure transitive ergative usuelle, paraphrase dans laquelle le syntagme nominal correspondant à notre sujet transitif est à l'absolutif, et non plus à l'ergatif. » (op. cit. 149). Un peu plus loin, l'auteur cite comme exemple conforme à la définition un énoncé appartenant au parler biscayen d'Oñate: umea liburua leiuta dao traduit par 'l'enfant "est ayant" lu le livre'.

Aussi peu que je me complaise dans le rôle plutôt ingrat du trouble-fête, il faudra quand même que je fasse ici une observation quelque peu critique. C'est que je me vois obligé de signaler qu'au lieu d'affirmer sans réserve "l'antipassif se définit... », l'aùteur eût fait mieux d'écrire: "je définis l'antipassif... ». En effet, la définition que je viens de citer et celle de Silverstein reprise par Dixon ne recouvrent pas du tout la même notion. Dans celle-ci, le syntagme absolutif figurant dans la structure ergative est censé se dégrader en cas oblique; dans l'exemple rebuschien, par contre, il persiste tel quel. De plus, alors que pour Silverstein et ses 
confrères il s'agit d'une diathèse, la définition rebuschienne fait appel à la notion de paraphrase, notion plutôt vague, qui, en outre, ne prévoit aucune limite au nombre de subordonnées qu'on puisse utiliser pour paraphraser une proposition. Ainsi, dans l'exemple cité, la paraphrase umea liburua leiuta dao de la phrase simple umeak liburua leiu dau 'l'enfant a lu le livre' n'a plus le statut d'une phrase simple: son verbe dao sert de copule et son prédicat liburua leiuta est un prédicat complexe, construit lui-même à partir d'une phrase.

Dans un livre remarquable portant le titre de Structure de l'énoncé en basque, le même auteur fait mention de deux catégories d'antipassifs: la première est constituée par des exemples tels que makina bat kaskarreko artuta nago 'je suis ayant reçu un tas de claques' (page 438); la seconde étant illustrée par zu niri liburuak emanda zaude 'vous êtes m'ayant donné les livres' (page 441). Il y aurait lieu, d'ailleurs, d'en distinguer encore une troisième: zu nik liburuak emanda zaude 'vous êtes celui à qui j'ai donné les livres'. En étudiant ces structures, l'auteur déclare sans ambages: "... l'antipassif basque s'oppose à celui du dyirbal, de l'esquimau et du quiché sur tous les points structuralement pertinents. " (page 447). Il continue: "faut-il pour autant nier l'existence d'un antipassif en euskara..? " A quoi il répond par la négative: "Nous ne le pensons pas,... parce qu'il nous semble injustifiable de ne pas vouloir chercher pourquoi les éléments de séries paraphrastiques telles que $(2 a-c)$ ou $(3 a-d)$ sont similaires, et en même temps en quoi ils s'opposent les uns aux autres" (p. 447). Bien entendu, je suis pleinement d'accord sur ce point. Je n'ai rien contre l'étude de telles structures. Elles font partie de l'économie de la langue et surviennent continuellement dans la pratique de la conversation courante. Aussi la question n'est-elle pas là. Tout ce que je soutiens, c'est que le nom donné à ces structures est mal choisi. Sur les traces de M. Rebuschi, M. Jean-Baptiste Coyos, l'auteur d'une étude capitale intitulée Le parler basque souletin des Arbailles explore des structures du type haurrak zopa janik dira 'les enfants sont ayant déjà mangé la soupe', structure qu'il a baptisée l'antipassif du parfait. Dans le même chapitre, il y joint un "antipassif du second prospectif" illustré par l'énoncé haurrak zopa jateko dira 'les enfants n'ont pas encore mangé la soupe', ou, comme il explique: 'les enfants sont pour action de manger la soupe' (op. cit. 369).

A lui aussi je voudrais lancer cet appel: De grâce, veuillez changer de terme en inventant une autre désignation, que ce soit parapassif, quasipassif, métapassif, ou je ne sais quoi, mais non antipassif, car ce terme-là, déjà breveté, ne se trouve plus disponible.

Soumise la requête, reposons notre question: $y$ a-t-il en basque un antipassif syntaxique?

Afin d'y répondre, je vais citer quatorze paires de phrases telles que le premier membre est une phrase transitive de structure ergative, tandis que le second membre est une phrase intransitive ayant même verbe, dans laquelle le syntagme correspondant à l'objet direct de la première phrase est marqué du cas instrumental à désinence $-z$ sans pour autant perdre son rôle de thème. Comme l'emploi de l'auxiliaire intransitif dans le second membre par opposition à l'auxiliaire transitif du premier s'interprète sans peine comme un marqueur de l'antipassif, rien ne s'opposera à considérer le second membre l'antipassif du premier. 
Une bonne partie des phrases utilisées a été empruntée au fonds de la littérature basque, tant ancienne que moderne. Dans ce cas, la provenance a été notée. Cette indication ne permet évidemment aucune conclusion sur le statut de la phrase dans l'usage contemporain. L'absence d'une telle indication, par contre, signale que la phrase est acceptable pour la plupart des locuteurs actuels. De toute façon, un commentaire succinct pour servir de guide au lecteur non initié sera suppléé au besoin.

La première paire qu'on va voir est axée sur le verbe ahaztu, 'oublier'. Compte tenu de la variation lexicale, comme l'emploi de ahantzi au lieu d'ahaztu, les deux tournures sont d'usage courant dans tous les dialectes actuels.

(1)a. Aspaldi ahaztu gintuen.

Il nous avait oubliés depuis longtemps.

(1)b. Aspaldi ahaztu zen gutaz. (Arana, Ipuin-entzulea 32)

Il nous avait oubliés depuis longtemps.

Puis nous pouvons citer le verbe baliatu 'employer', qui, dans son emploi intransitif se traduit généralement par 'se servir de'.

(2)a. Ez dugu hitz hori baliatzen. (G. Garate, Erdarakadak 38)

Nous n'employons pas ce mot.

(2)b. Ez gara hitz horretaz baliatzen.

Nous ne nous servons pas de ce mot.

La tournure intransitive est beaucoup plus usuelle ici que la transitive, qui survit cependant dans la langue standard et figure chez les meilleurs écrivains, tels que, par exemple, Etcheberri de Ciboure, Axular, Elissamburu, Duvoisin, Saint-Pierre, Hiribarren, Jean-Baptiste Etchepare, Narbaitz, Xalbador et Lafitte.

Continuant l'ordre alphabétique, voici le verbe burlatu 'se moquer':

(3)a. Buruzagiek burlatzen zuten Jesus. (Luc 23.35; Duvoisin)

Les chefs raillaient Jésus.

(3)b. Buruzagiak burlatzen ziren Jesusez.

Les chefs se moquaient de Jésus.

De par son origine romane, ce verbe est assez peu employé de nos jours. La structure transitive, qui est aujourd'hui la moins usitée, est attestée chez Etcheberri de Ciboure, Maister, Duvoisin, Uriarte, Xenpelar, Laphitz et Lapeyre. Chez celui-ci on rencontre les deux toumures: Erlijionea eta haren mixterio sainduak burlatzen dituztenak (Kredo 59) 'ceux qui raillent la religion et ses mystères saints', et aussi ez naiz zutaz burlatzen (Kredo 59) 'je ne me moque pas de vous'.

$\mathrm{Au}$ sens d'enfanter, erditu et erdi sont des variantes dialectales occupant le même lemme dans le dictionnaire (Cf. $D G V$ VI, 811). La tournure intransitive est la plus fréquente, mais la transitive est attestée chez Cardaveraz, Uriarte, Ibiñagabeitia, Orixe et Zaitegi.

(4)a.Erdituko du semea. (Mt. 1.21; Uriarte)

Elle enfantera un fils. 
(4)b.Erdiko da seme batez. (Mt. 1.21; Duvoisin)

Elle enfantera un fils.

De nos jours, le verbe errukitu 'avoir pitié' est généralement employé comme intransitif. Mais l'exemple transitif (5)a est jugé admissible dans le dictionnaire normatif d'Tbon Sarasola.

(5)a.Zeuon buruak errukitu itzazue. (Guerrico, I, 438)

Ayez pitié de vous-mêmes.

(5)b.Zeuon buruez errukitu zaitezte.

Ayez pitié de vous-mêmes.

Eskarniatu 'se moquer' est un verbe archaïque qui ne s'emploie plus aujourd'hui. La tournure transitive se trouve chez Oihenart, Etcheberri de Ciboure et Liçarrague: Eta hek eskerniaturen dute hura (Mc. 10.34) 'Et ils se moqueront de lui'.

(6)a. Hori da Jainkoa eta Jainkoaren miserikordia eskarniatzea.

C'est se moquer de Dieu et de la miséricorde de Dieu.

(6)b.Hori da Jainkoa eta Jainkoaren miserikordiaz eskarniatzea. (Axular, Guero, 150)

C'est se moquer de Dieu et de la miséricorde de Dieu.

Le verbe gogoratu 'se rappeler' s'emploie toujours dans les deux tournures:

(7)a.Grogora (ezazue) Lot-en emaztea. (Luc. 17.32)

Rappelez-vous la femme de Lot.

(7)b.Gogora zaitezte Lot-en emazteaz.

Souvenez-vous de la femme de Lot.

Dans le sens de 'jouir', le verbe gozatu admet toujours les deux tournures. Les deux exemples qui suivent sont en dialecte souletin:

(8)a. Gozatüren düzü barnetiko bake handia.

Vous jouirez d'une grande paix intérieure.

(8)b. Gozatüren zira barnetiko bake handiaz. (Maister, Imit. III, 37.3) Vous jouirez d'une grande paix intérieure.

Le même auteur utilisait aussi la tournure transitive, témoin sa traduction d'une autre phrase du même texte: pacificam mentem possideamus, qui est rendue par arimaren bakia goza dezagün (Imit. I, II, 4) 'jouissons de la paix de l'âme'.

La tournure antipassive existe également pour le verbe hautatu 'choisir':

(9)a. Aziendek orobat hautatzen dute bazka hobeena.

Les animaux choisissent de même la meilleure nourriture.

(9)b. Aziendak orobat hautatzen dira bazka hobeenaz. (Duvoisin, Laborantzako liburua, 264) 
Les animaux choisissent de même la meilleure nourriture.

Bien qu'absente dans le Guero d'Axular, la tournure antipassive du verbe hautatu est abondamment attestée dès le début de la littérature basque. C'est précisément un verset de Bernard d'Etchepare, le premier poète basque à se faire imprimer, qui a fourni l'inspiration pour mon article: "Andre eder gentil batez hautatu zait begia » (XII-1), c.-à-d. 'Mon oeil a choisi une belle et gentille dame'. Et dans le Nouveau Testament de Liçarrague: ... nola lehen jarlekhuéz hautatzen ziraden (Luc. 14.7), '... comment ils choisissaient les premières places'. Aux deux exemples extraits du livre Noelak d'Etcheberri de Ciboure par le Diccionario General Vasco (III, 451), on peut en ajouter un troisième: ... eta zeruko ontasun fin gabeez hautatu (p. 171) 'et (vous avez) choisi les richesses sans fin du ciel'. Le dictionnaire cité signale aussi des exemples postérieurs: dans la tragédie du Charlemagne, chez Monho, Hiribarren, et surtout, Duvoisin. Dans son dictionnaire manuscrit, le capitaine observe: On dit " hauta zazu nahi duzuna " ou " hauta zaite nahi duzunaz » (c-à-d. 'choisissez ce que vous voulez').

A présent, d'après une enquête réalisée par l'auteur de ces lignes auprès des académiciens basques, l'antipassif de ce verbe est entièrement sorti de l'usage.

Dans une perspective diachronique, le verbe mendekatu 'se venger' n'est pas dénué d'intérêt. L'exemple transitif ci-dessous a été emprunté au père jésuite Bernard Gasteluçar, qui naquit à Ciboure en 1614. L'exemple figure dans son ouvrage Eguia catholicac, paru en 1686.

(10)a. Zuk mendekatuko tutzu gizon guztien etsaiak. (Gasteluçar, 65)

Vous tirerez vengeance des ennemis de tous les hommes.

(10)b. Zu mendekatuko zara gizon guztien etsaiez.

Vous vous vengerez sur les ennemis de tous les hommes.

Il faut préciser que la construction illustrée par (10)a ne subsiste plus à l'heure actuelle. La même formule énoncée aujourd'hui aurait un sens radicalement différent, à savoir 'vous vengerez les ennemis de tous les hommes'. Et notez bien que (10)b ne représente plus l'antipassif de (10)a si celui-ci est compris dans le sens moderne. Rappelons en effet que, pour qu'on puisse parler d'antipassif, il est essentiel que le rôle thématique qu'assume le syntagme instrumental dans la tournure intransitive soit identique à celui du syntagme absolutif correspondant dans la structure transitive. Or, il est évident que l'absolutif gizon guztien etsaiak 'les ennemis de tous les hommes' dans (10)a au sens actuel et l'instrumental correspondant dans $(10) \mathrm{b}$, remplissant des fonctions sémantiques différentes, ne partagent pas le même rôle thématique.

A vrai dire, en ce qui concerne le propos de cet article, tout ceci n'a aucune importance, car la structure intransitive au verbe mendekatu ne laisse pas d'être un antipassif d'origine, bien que ne l'étant plus sur le plan synchronique.

Pour ce qui est du verbe mintzatu 'parler', l'usage du cas instrumental pour désigner la langue suggère une origine antipassive:

(11)a. Hiruek mintzatzen dute eskuara. (J. Etchepare, Beribilez, 86) Les trois parlent le basque.

(11)b. Hiruak mintzatzen dira eskuaraz. 
Les trois parlent (en) basque.

Pour le verbe nagusitu 'dominer', on peut citer: 521)

(12)a. Zer pasionek gure bihotza nagusitzen dute? (Cf. S. Pouvreau, Philotea, Quelles passions dominent notre coeur?

(12)b. Zer pasione gure bihotzaz nagusitzen dira?

Quelles passions s'emparent de notre coeur?

Le verbe oroitu 'se rappeler' s'emploie encore couramment dans les deux structures:

(13)a. Oroitzen zituen ume-umetatik Malentxoz edukitako arretak. (Agirre, Garoa, 243)

Il se rappelait les soins qu'il avait prodigués à Malentxo dès son enfance.

(13)b. Oroitzen zen ume-umetatik Malentxoz edukitako arretez. enfance.

Il se souvenait des soins qu'il avait prodigués à Malentxo dès son

Le verbe trufatu 'se moquer', emprunt au béarnais trufà de même sens, connaît les deux structures:

(14)a. Nork trufatu zaitu berriz ere? (Lafitte, Murtuts 41)

Qui s'est payé ta tête encore?

(14)b. Nor trufatu da zutaz berriz ere?

Qui s'est payé ta tête encore?

Ce verbe a été classé comme intransitif par M. B. Oihartzabal dans son essai "Structural Case and Inherent Case Marking: Ergaccusativity in Basque", voir la page 335. Il faut pourtant remarquer que la tournure transitive est attestée chez pas mal d'écrivains: Argaignarats, Baratciart, Duvoisin, Laphitz, Hiribarren, l'auteur du catéchisme publié par Jauffret, J. Etchepare, Etchamendi et Lafitte.

La parade d'exemples terminée, que concluera-t-on? On peut certes soutenir que l'alternance antipassive telle que nous l'avons observée a l'air d'être un phénomène purement lexical. C'est d'ailleurs l'avis du professeur J. Ortiz de Urbina qui s'est avisé le premier de cette alternance et l'a rapprochée, non sans hésitation, de l'antipassif de certaines langues. Voici ce qu'il en dit à la page 203 de son livre Parameters in the Grammar of Basque, publié en 1989:

"This is the 'antipassive' construction often encountered in ergative languages. There is no equivalent structure in Basque, although lexically some verbs may appear in two structures that are closely parallel to the structures found in the ergative-antipassive relation. Thus, a verb like oroitu 'to remember' may appear in a transitive structure with absolutive direct object and ergative subject or 
in an unaccusative one where the subject is marked absolutive and the object occurs in the oblique instrumental case.

(i)

a. Familia oroitu zuen

He remembered his family

b. Familiaz oroitu zen

He remembered his family

Similar lexical relations are found in non-ergative languages. "

Effectivement, pour ce qui est de la langue actuelle, je suis parfaitement d'accord sur le point de vue qu'on vient de lire. Mais, en remontant le fil du temps? Comme les verbes admettant la tournure antipassive sont de nature assez diverse, et que plusieurs, tels qu'erditu 'enfanter' et hautatu 'choisir', manquent de tout modèle roman, il me semble plus que probable que, du point de vue diachronique, nous avons affaire à des reliques d'un procès qui, du moins à l'origine, relevait bien de la syntaxe. En bref, j'estime que les faits que nous venons d'observer justifient de postuler un véritable procédé antipassif pour le basque ancien, disons du premier millénaire.

A l'encontre de la situation observée dans l'idiome dyirbal de l'Australie, il est peu probable que l'antipassif basque pût s'appliquer à toutes les phrases transitives sans égard à la nature du verbe. Nos données nous permettent d'entrevoir plutôt que le procédé était limité à une certaine classe de verbes, les verbes dits "moyens".

A ce propos, il convient de rappeler la caractérisation formulée par le savant éminent que fut $\mathrm{E}$. Benveniste; formulation dont l'état actuel de notre science ne permet toujours pas de remédier à l'imprécision: Dans le moyen, le verbe indique un procès dont le sujet est le siège; le sujet est intérieur au procès " (Problème de linguistique générale, p. 172).

Or, il faut relever que cette caractérisation s'applique de façon frappante à tous les exemples que nous avons vus. Ainsi, procédant par induction, j'arrive à la conclusion que c'est précisément l'ensemble des verbes moyens qui constitue le domaine de l'antipassif en basque ancien. Un tel résultat ne manque pas d'implications diachroniques. Ce qu'on peut constater, par exemple, c'est que pour certains verbes la toumure antipassive a fini par évincer la tournure transitive originelle. Cela semble bien être le cas des verbes moyens fidatu 'se fier' et jabetu 's'approprier'. Si, comme j'estime, ces verbes étaient originellement transitifs, la présente agrammaticalité de phrases comme ${ }^{*}$ fidatzen zaitut et ${ }^{*}$ etxe horiek jabetuko ditugu montre qu'ici l'antipassif a réussi à s'imposer obligatoirement, fournissant les phrases zutaz fidatzen naiz 'je me fie à vous' et etxe horietaz jabetuko gara 'nous nous emparerons de ces maisons'. Ajoutons que l'emploi transitif de jabetu est attesté. Le Diccionario General Vasco cite une phrase de Kepa Enbeitia avec jaubetu, variante biscayenne de jabetu: Zetan ez doguz jaubetu biar? 'Pourquoi ne les approprierions-nous pas?'.

D'une façon générale, je dirais qu'il y a lieu de soupçonner une origine antipassive chaque fois qu'un syntagme instrumental sert de thème dans une structure intransitive. La condition que je viens de formuler est nécessaire. En effet, si le syntagme instrumental ne sert pas de thème, il ne saurait s'agir d'un antipassif.

En guise d'illustration, j'invoquerai ici les verbes indiquant un état de l'âme. Ces verbes présentent une alternance systématique due au fait que la source de 
L'émotion dénotée par le verbe se réalise soit par un syntagme à l'ergatif, soit par un syntagme à l'instrumental. On a donc:

(15)a. Gure alabek kezkatzen naute. Mes filles m'inquiètent.

(15)b. Gure alabez kezkatzen naiz. Je m'inquiète de mes filles.

(16)a. Zure portaerak harritzen gaitu.

Votre conduite nous étonne.

(16)b. Zure portaeraz harritzen gara.

Nous nous étonnons de votre conduite.

(17)a. Miren zoriontsu ikusteak pozten nau.

Ca me réjouit de voir Marie heureuse.

(17)b. Miren zoriontsu ikusteaz pozten naiz. Je me réjouis de voir Marie heureuse.

Comme le rôle thématique du syntagme instrumental dans ces exemples est celui de source et non pas de thème, nous prédisons qu'il ne peut s'agir d'une alternance antipassive. C'est exact, car, pour qu'il y ait antipassif, il faudrait que le rôle thématique de l'instrumental coïncide avec celui de l'absolutif dans la tournure transitive. Or, il n'en est rien; c'est, au contraire, l'ergatif qui a prêté son rôle au syntagme instrumental.

Nous avons maintenant tout ce qu'il faut pour passer à la partie finale de cet essai, dans laquelle, en examinant les verbes intransitifs du basque, nous essaierons de mettre à profit les connaissances acquises sur l'antipassif.

\section{Deuxième partie: l'hypothèse de Levin}

Depuis l'article pionnier "Impersonal Passives and the Unaccusative Hypothesis" de D.M. Perlmutter, on sait que les verbes intransitifs se divisent en deux classes, pour lesquelles, à tort ou à raison, on a adopté les termes d'inaccusatif et d'inergatif.

Comme définition de ces termes, je propose la suivante: $\mathrm{Si}$, à un certain niveau d'analyse, le seul ou principal actant du verbe est interne au prédicat, il s'agit structuralement d'un objet direct, et le verbe se classifie comme inaccusatif; sinon, le verbe comportera un sujet à tous les niveaux d'analyse, et se classifie comme inergatif.

La différence structurale sur laquelle se fonde la définition comporte des répercussions syntaxiques variées, comme l'ont montré B.C. Levin et M. Rappaport Hovav dans leur monographie exemplaire intitulée Unaccusativity. Pour ne prendre qu'un exemple, le participe parfait d'un verbe inaccusatif peut parfois servir d'adjectif; celui d'un verbe inergatif n'a jamais cette option. On dit donc bien emakume eroria 'la femme tombée', mais non pas *emakume borrokatua '*la femme luttée'. Dans l'optique de Perlmutter, la divergence structurale entre les deux classes n'est pas arbitraire. Elle est déterminée par le sens du verbe. 
Seulement, dans sa tentative de caractérisation sémantique des verbes inergatifs, Perlmutter s'en est tenu au critère trop simpliste d'agentivité, critère dont on a vite fait de découvrir l'insuffisance.

Comme l'ont montré Levin et Rappaport Hovav, il y a des catégories de verbes où le sujet contrôle l'action et qui sont néanmoins inaccusatifs. Tel est le cas notamment de la catégorie qu'elles caractérisent comme des verbes de motion à direction inhérente; catégorie contenant toute une gamme de verbes intransitifs tels que, par exemple, arriver, avancer, échapper, entrer, fuir, partir, plonger, sortir, tomber et venir.

Bien que pour la plupart d'entre eux le sujet du verbe ait plein pouvoir sur le déroulement de l'action, ces verbes n'en sont pas moins tous inaccusatifs.

Je n'ai pas le dessein de creuser la théorie de l'inaccusativité, tout fascinante qu'elle est. Le lecteur curieux des propriétés syntaxiques des verbes inaccusatifs ainsi que des critères sémantiques à utiliser pour effectuer le triage consultera la monographie citée ci-dessus.

Ce qui nous intéresse tout particulièrement en tant que basquisants, c'est que Mme Levin, à la suite d'une étude poussée des faits basques, a hasardé l'hypothèse que tous les verbes basques excluant l'ergatif font partie de la catégorie inaccusative.

Cette hypothèse osée, et d'une portée considérable, comme on s'en apercevra par la suite, a été récusée par certains spécialistes, notamment par le grammairien B. Oihartzabal. A mintzatu 'parler', l'unique exception signalée par Mme Levin, ce linguiste, locuteur natif du basque labourdin, en a ajouté beaucoup d'autres. Je cite: "In fact, there are many other exceptions: other speech verbs (solastatu 'speak with', elekatu, elestatu 'chat', hizkatu, hizketatu 'converse'), meal verbs (bazkaldu 'have lunch, afaldu 'have dinner', gosaldu 'have breakfast', askaldu 'have a snack'), several other verbs having agentive arguments (trabailatu 'work', jarraiki 'follow $(s b)$ ', mendekatu 'take revenge', jazarri 'revolt', oldartu 'attack', gudukatu 'wage war on', borrokatu 'fight', jostatu 'play', jokatu 'to ploy games', etc...). » (Oihartzabal, "Structural Case and Inherent Case Marking: Ergaccusativity in Basque ", p. 335). Et au bas de la même page, à côté des locutions trufa egin "se moquer' et zintz egin 'se moucher', l'auteur signale encore les verbes synonymes trufatu et zintzatu.

Il y a lieu d'interposer une question. Est-il vrai que tous les verbes cités sont inergatifs? M. Oihartzabal invoque bien la nature agentive de leurs sujets, mais comme nous venons de le voir, ce critère n'est pas suffisant, notamment dans le cas de verbes de motion à direction inhérente. Et en fait, je suis d'avis que la liste d'Oihartzabal inclut deux verbes qui sont d'excellents candidats pour cette catégorie-là. Il s'agit du verbe jarraiki 'suivre', et aussi du verbe oldartu, dont la glose 'attaquer' ne convient ni au sens originel ni au sens en labourdin actuel, qui est plutôt 'prendre l'élan'. Je reviendrai ci-dessous sur ces verbes.

Pour le moment, tout cela ne semble pas revêtir trop d'importance, car les autres verbes dont il est question appartiennent bel et bien à la catégorie inergative. Ces verbes, manquant d'actants ergatifs, s'érigent en d'authentiques contre-exemples à l'hypothèse de Levin, qui se voit donc réfutée.

Mais attention! N'oublions pas de préciser qu'elle se trouve réfutée pour le labourdin actuel et qu'il ne s'ensuit nullement qu'il en fût de même à une période 
plus ancienne. Et voilà justement l'objectif des pages qui suivent. En effet, je vise à démontrer ici la validité de l'hypothèse de Levin pour un basque plus ancien, disons jusqu'au seizième siècle environ.

A cette fin, il faudra montrer que les verbes inergatifs du basque contemporain n'existaient pas comme tels dans un passé plus ou moins proche. Plus exactement, ce que je ferai valoir dans mon exposé, c'est ceei; Certains verbes inergatifs sont devenus tels à la suite d'un changement de sens, tandis que les autres doivent leur origine à un verbe transitif, dont la tournure transitive est tombée en désuétude, le plus souvent dans une partie seulement du territoire bascophone.

Je commencerai la démonstration par le verbe jarraiki 'suivre'. Comme j'ai déjà remarqué, je crois que c'est à tort que $M$. Oihartzabal l'a qualifié d'inergatif. Dans son sens principal, il s'agit d'un verbe de motion à direction inhérente, catégorie inaccusative d'après les recherches de Levin et Rappaport Hovav. Rien de surprenant donc que ce verbe ait été intransitif à l'origine, comme le montrent les textes anciens, labourdins aussi bien que biscayens, et, avant tout, la structure à préfixe personnel des formes synthétiques: arreit niri 'suis-moi' (MC. 9.9; Liç.) et inurriari arraio (R.S. 183) 'suis la fourmi'.*

Ce n'est que plus tard que surgissent les formes transitives, et -ce qui est révélateur- d'abord dans des sens secondaires. Ainsi Belapeyre n'use de la construction transitive que lorsque le verbe veut dire 'imiter': p. ex. lürrian ere hain onsa zure honkiginak eta bizitze saintia egizü jarraik ditzadan (Cat. I, 137) 'faites que j'imite tant bien sur la terre aussi vos bonnes actions et votre vie sainte'; haren verthüter jarraiki ditian (Cat. II, 109), 'qu'il imite ses vertus'.

En ce qui concerne le verbe oldartu, si on lui donne le sens d'attaquer, il est inergatif. Seulement, ce n'est pas le sens qui lui correspond à l'origine. Dans le dictionnaire de Lhande, le sens qui est indiqué le premier, c'est "s'élancer, se précipiter, se jeter sur". Ce sens-là est aussi signalé par Villasante chez Axular. Et, témoignage plus important encore parce que plus ancien, il suffit de scruter l'emploi du terme chez Liçarrague, où le verbe se rencontre plus d'une douzaine de fois, pour constater que son sens était 'se lancer', plutôt que celui d'attaquer. Par conséquent, c'était un verbe de motion à direction inhérente, et, dès lors, inaccusatif.

Quant au verbe trabailatu, au sens de travailler, il est indubitablement inergatif, comme le montre clairement la locution synonyme lan egin. Mais est-ce là son sens primitif? Pour trouver la réponse, il nous faut retourner à l'oeuvre de Liçarrague. Dans sa traduction du Nouveau Testament, le verbe en question figure à deux sens, assez proches l'un de l'autre, mais quand même différents. D'une part, il sert à

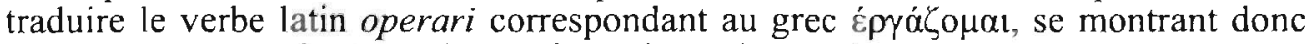
l'équivalent du verbe travailler en français moderne. Ceci est le cas dans les versets Mt. 21.28; Jn. 6.27; I Cor. 4.11; Eph. 4.28; Col. 1.29; 2 Thes. 3.10 et 2 Thes. 3.12. D'autre part, trabailatu sert de traduction pour le verbe latin laborare, correspondant au grec колíaw, dont l'équivalent français est 'se donner de la peine', 'se fatiguer'. Les versets pertinents sont: Actes 20.35; Rom. 16.6; Rom. 16.12; Gal. 4.11; Eph. 4.28; Col. 1.29; 1 Thes. 5.12; l Tim. 5.17; 2 Tim. 2.6. Remarquons que dans les évangiles, laborare se rend toujours par nekhatu: Mt. 6.28; Luc 5.5; Luc 12.27; Jn. 4.38. 
Or, il est évident que l'évolution sémantique qui mène du sens 'se fatiguer' à celui de travailler est beaucoup plus probable que l'inverse.

On sait du reste que le verbe travailler en français a subi une évolution parallèle. Je cite du Nouveau dictionnaire étymologique du français de Jacqueline Picoche: "Travailler XII s. 'tourmenter' et 'souffrir', début XVY's. sens mod. (à partir de la forme pronom. 'se donner de la peine pour') 》 (p. 487).

Il se trouve donc que le verbe trabailatu, avant de prendre le sens moderne de 'travailler', servait d'équivalent à nekatu 'se fatiguer'. Comme lui c'était donc un verbe de changement d'état, et, par conséquent, appartenait à la catégorie inaccusative. Citons, à ce propos, Levin et Rappaport Hovav: "The unaccusative status of verbs of change of state, especially those that participate in the causative alternation, has been assumed by linguists working on unaccusativity beginning with Perlmutter (1978), who included them among the semantic classes of unaccusative verbs on the basis of their behavior with respect to impersonal passivization. In Italian these verbs pass the standard unaccusative tests, including selection of the auxiliary essere 'be'. In English these verbs can appear in the unaccusative resultative pattern and cannot assign accusative Case, as shown by their inability to take various types of nonsubcategorized objects." " (Unaccusativity, 293). Le cas du verbe ausartu 'oser', soit dit en passant, est tout à fait semblable. D'origine, c'est un verbe dérivé de l'adjectif ausart 'hardi', verbe dont l'emploi transitif 'enhardir' est attesté chez Axular (p. 450). Tout comme nekatu, c'est un verbe de changement d'état participant à l'alternance causative, et, par conséquent, il est inaccusatif.

Quant aux autres verbes cités en contre-exemple par M. Oihartzabal, je vais montrer qu'il s'agit de verbes transitifs à l'origine, qui ont perdu leur emploi transitif en labourdin actuel.

Pour deux de ces verbes la démonstration en a déjà été faite. Je me réfère aux verbes mendekatu 'se venger' et trufatu 'se moquer', dont l'emploi intransitif s'est révélé être un antipassif, comme on l'a vu dans la première partie de cet essai.

Le verbe zintzatu 'se moucher' ne pose pas de problème non plus. Je veux bien croire qu'il est intransitif en labourdin actuel, mais non pas qu'à aucune époque on n'ait pu dire l'équivalent basque de la phrase française la mère va moucher ses enfants. Du point de vue diachronique, l'intransitif zintzatu, comme se moucher en français, n'est autre que la forme médio-réflexive du transitif zintzatu 'moucher'.

Disons maintenant quelques mots sur les verbes de la parole. Dans la première partie de cet essai, nous avons pu constater l'emploi transitif du verbe mintzatu avec un nom de langue servant d'objet direct: eskuara mintzatu 'parler le basque'. Il faut cependant ajouter que l'emploi transitif de ce verbe s'étend bien au-delà de cette structure. Dans le basque du sud notamment, cet emploi a été si répandu que, très souvent, il faut traduire par dire plutôt que par parler. En voici quelques exemples: nori mintzatzen diozu (Añibarro, Cat. AN 33), 'à qui le dites-vous?'; danak zerbait mintzatzen ziotela (Agirre, Garoa 335) 'que tous lui disaient quelque chose'; itz oek mintza bitartean ( $\mathrm{T}$. Agirre, Uztaro 306), 'en disant ces mots'; zuk itz-erdi bat mintza orduko (S. Mitxelena, Aranz. 62), 'dès que vous dites un demi-mot'; erlojuen gainean ez ginuen deus mintzatu (Izeta, Dirua G. 84), 'sur la montre nous avons rien dit'. 
Le même usage est attesté en labourdin: hitzik mintzatu gabe (Etcheberri de Sare, 384), 'sans dire un mot'.

Dans les parlers septentrionaux, on rencontre aussi une construction transitive dans laquelle la personne à qui l'on parle sert d'objet direct. Pour la documentation, je renvoie au Diccionario General Vasco (XII, 369), où l'on trouvera des exemples empruntés à une dizaine d'auteurs, labourdins pour la plupart.

Pour les autres verbes de la parole, la même construction existe, bien qu'elle soit moins usitée. Le dictionnaire cité la signale pour les verbes elestatu et elekatu, et pour solastatu 'converser', on peut citer un exemple de Pouvreau: zergatik ordea hain gogotik solhastatzen dugu elkar? (Imit. I. 10,1), 'mais pourquoi nous parlonsnous si volontiers l'un à l'autre?' (L'original latin dit: invicem fabulamur). On constate donc que l'emploi de ces verbes a été possible partout où ils existaient, y compris le labourdin. Du point de vue diachronique, on soutiendrait donc mal que les verbes de la parole eussent exclu l'ergatif.

Quant aux verbes relatifs aux repas, leur emploi transitif est abondamment attesté. Déjà chez le frère Ubillos on lit: Cer zan Bazcoa? Israeltarrak Igitotik irten ciran gau artan afaldu zuen vildotsa (Christau doc. 24), 'Qu'est-ce que la Pâque? L'agneau que (c.-à-d. duquel) soupèrent les israélites la nuit qu'il quittèrent l'Egypte'. Et toujours, chez Lopez-Mendizabal: Zer nai dezute gosaldu? (Manual de Conv. 190), 'Que voulez-vous manger pour le petit-déjeuner?'.

Dans les parlers du nord, l'emploi comportant un objet direct s'est perdu très tôt, ce qui a abouti à une détransitivisation, qui peut fort bien se concevoir comme l'effet d'un procédé antipassif, d'application obligatoire si le syntagme absolutif est vide de contenu.

Dans les parlers du sud, au contraire, ces verbes continuent à exiger l'ergatif même si aucun objet direct n'est envisagé. Donc: bazkaldu dugu 'nous avons déjeuné'.

Point n'est besoin de nous attarder sur les verbes jostatu 'amuser' et jokatu 'jouer'. Il suffit de consulter le Diccionario General Vasco (X, 257-259 et 281-282) pour vérifier que leur emploi transitif est attesté tant au nord comme au sud.

J'en viens maintenant aux verbes borrokatu 'lutter' et gudukatu 'combattre'. Ici la documentation dans le Diccionario General Vasco est très révélatrice et permet de conclure qu'il s'agit de verbes transitifs qui peuvent prendre un objet direct, mais le plus souvent, n'en prennent pas. Dans ce cas-là, ils tendent à emprunter la tournure intransitive, ce qui, dans certains parlers du nord, peut avoir abouti à l'obsolescence de la tournure transitive. Ici encore, la détransitivisation amenée par l'absence d'un objet suggère l'application de l'antipassif.

Pour ce qui est du verbe jazarri, il suffit de citer la Grammaire basque de P. Lafitte: "Les anciens construisaient comme behatu les verbes iguriki 'attendre"; jazarri 'attaquer'; lagundu 'aider'. Ex.: etsaiek Jesusi iguriki zioten, jazarri zioten eta nihork ez zion lagundu 'les ennemis attendirent Jésus, l'attaquèrent, et personne ne le secourut'. » (Lafitte, Grammaire basque § 412, p. 190).

L'exemple qu'on vient de lire mérite d'ailleurs l'attention, car il montre que le labourdin ancien admettait des phrases transitives où ne figure aucun objet direct.

Ceci enfin complète l'examen des contre-exemples cités par M. Oihartzabal. A mon avis, nous avons réussi à les disqualifier tous. Dans un passé plus ou moins 
proche, les candidats ou bien se montraient inaccusatifs ou bien n'étaient que des emplois intransitifs de verbes foncièrement transitifs.

Je reconnais que pour une vérification rigoureuse de l'hypothèse en question il faudrait examiner les verbes inergatifs du basque dans leur intégralité. Cependant le lecteur me pardonnera de ne pas m'engager sur cette voie. D'après mon opinion, ce que nous avons pu observer suffit déjà pour justifier l'adhésion à cette hypothèse, toujours en ce qui concerne le basque ancien, disons jusqu'au début du seizième siècle.

S'il en est ainsi, quelles sont les conséquences qui en déroulent?

Commençons par le système des cas. Dans une langue où se vérifie l'hypothèse de Levin, l'attribution des cas grammaticaux aux actants du verbe présente un caractère des plus simples: L'actant extérieur au prédicat sera au nominatif, et l'actant intérieur au prédicat à l'accusatif.

Voilà exactement ce qui se passe en basque ancien, sauf que, pour des motifs se rapportant à la langue moderne, l'accusatif et le nominatif s'appellent absolutif et ergatif. Mais les noms ne changent rien à la chose: le basque ancien n'était nullement une langue ergative.

Typologiquement parlant, le basque ancien rejoint l'ancien français. Les deux langues ont un accusatif à marque zéro ainsi qu'un nominatif marqué de façon positive: $-s$ en ancien français; $-k$ en basque ancien. Il reste pourtant une différence fondamentale. En ancien français, le sujet d'un verbe inaccusatif est au nominatif, tandis qu'en basque, il demeure à l'accusatif, même s'il lui arrive d'être extrait du prédicat dans la structure superficielle.

L'hypothèse de Levin acceptée comme étape dans l'histoire du basque, on est en mesure d'expliquer bien des caractéristiques actuelles, qui, sans elle, resteraient inexpliquées, voire inexplicables.

Pour commencer, considérons la pénurie dans le domaine des verbes intransitifs comparée au français. Elle est facile à expliquer: en français, il y a des intransitifs tant inaccusatifs qu'inergatifs; en basque, il n'y a que les seuls inaccusatifs.

Autre fait typique: Au petit nombre de locutions idiomatiques avec le verbe faire du type faire dodo 'dormir', le basque en oppose plus de deux cents. C'est qu'en basque cette locution représente la manifestation préférée des verbes inergatifs, lesquels exigent un sujet ergatif, qui est fourni par cette construction transitive.

Les verbes que le grammairien P. Lafitte a qualifiés de déponents (Grammaire basque, $\S 411$, p. 189) ne constituent qu'un expédient de plus employé par la langue pour résoudre le problème des intransitifs inergatifs. Il s'agit là de verbes qui, tout en n'admettant aucun objet direct, requièrent quand même l'ergatif. Dans une langue ergative canonique, c'est là un paradoxe assez pénible. Dans le basque ancien, par contre, il n'était que naturel que le sujet d'un verbe inergatif fût au nominatif, précurseur de l'ergatif moderne.

Pour beaucoup de verbes inergatifs, la langue a eu recours aux deux solutions à la fois. Prenons, par exemple, les verbes d'émission, catégorie inergative par excellence. Alors, d'une part, on trouve des locutions à base du verbe egin: burunba egin 'bourdonner', dirdira egin 'briller', distira egin 'étinceler'; de l'autre, il y a les verbes déponents: burunbatu 'bourdonner', dirdiratu 'briller', distiratu 'étinceler'.

Du moment que le basque actuel permet des verbes inergatifs à sujet absolutif, tous ces phénomènes ont perdu leur raison d'être. Ce sont des vestiges d'un état 
plus ancien, d'une époque où l'hypothèse de Levin rendait compte de la structure de la langue.

A présent, pour finir sur un thème abordé au début de cet article, retournons un moment à l'antipassif. J'ai affirmé au début de cet essai que c'était un procédé réservé aux langues ergatives. Pourtant, si l'évolution typologique que j'ai esquissée correspond tant soit peu à la réalité du basque, il faut constater ceci: Comme il ressort de mon exposé, aux temps où l'antipassif fonctionnait comme un authentique procédé de syntaxe, le basque n'était pas une langue ergative. Il ne l'est devenu qu'au fur et à mesure que l'antipassif en tant que procédé syntaxique est sorti de l'usage.

C'est un paradoxe évident, mais aussi un paradoxe facile à dissiper. Il n'existe, au fond, aucune raison valable pour croire à une limitation de l'antipassif aux seules langues ergatives. Dès lors, à côté du schéma élaboré ci-dessus pour ces langues, il faudra admettre également le schéma suivant:

ANTIPASSIF:

Comparons une fois de plus l'antipassif au passif:

PASSIF:

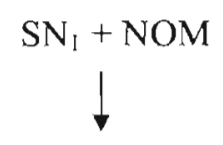

CAS OBLIQUE

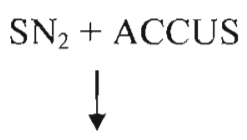

$\mathrm{SN}_{2}+\mathrm{NOM} \ldots$

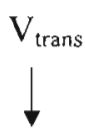

$V_{\text {pass }}$

J'espère que ces représentations schématiques aideront le lecteur à discerner en quoi l'antipassif ressemble au passif et en quoi ils s'opposent. Il s'agit de savoir, en somme, pour quel motif on s'est accordé sur le terme d'antipassif. C'est ainsi que je résumerais la comparaison: Dans le passif, le nominatif est rétrogradé en syntagme adverbial, mais l'accusatif, à l'inverse, est promu et devient nominatif.

Dans l'antipassif, par contre, il y a rétrogradation générale: le nominatif se dégrade en accusatif en même temps que l'accusatif se trouve rétrogradé en syntagme adverbial.

\section{Bibliographie}

Benveniste, Emile, "Actif et moyen dans le verbe", Journal de psychologie, XLIII, 121-129, 1950.

Benveniste, Emile, Problèmes de linguistique générale, Paris, 1966.

Bright, William, International Encyclopedia of Linguistics, New York / Oxford, 1992.

Cook, Eung-Do et Gerdts, Donna B., Syntax and Semantics: The Syntax of Native American Languages 16, Orlando etc. 1984.

Coyos, Jean-Baptiste, Le parler basque souletin des Arbailles, Paris, 1999. 
De Rijk, Rudolf P.G., “'Nunc' vasconice”, ASJU XXVI-3 (1992), 695-724 (inclu dans le numéro suivant).

De Rijk, Rudolf P.G., De lingua vasconum: selected writings, Bilbao, 1998.

Dixon, Robert M.W., The Dyirbal Language of North Queensland, Cambridge, 1972.

Dubois, Jean, et al., Dictionnaire de linguistique, Larousse, Paris, 1973.

Ducrot, Oswald et Schaeffer, Jean-Marie, Nouveau dictionnaire encyclopédique des sciences du langage, Paris, 1995.

Heath, Jeffrey, "Antipassivization: a Functional Typology", Proceedings of the Second Annual Meeting of the Berkeley Linguistics Society, 202-211, 1976.

Kuryłowicz, Jerzy, "Ergativnost i stadial'nost v jazyke", Izvestija Akademiji Nauk SSR, V 387-394, 1946.

Kuryłowicz, Jerzy, "La construction ergative et le développement stadial du langage", Annali della Scuola Normale Superiore di Pisa, XVIII, 84-92, 1949.

Lafitte, Pierre, Grammaire basque (navarro-labourdin littéraire), Bayonne, 1962.

Levin, Beth C., "Unaccusative verbs in Basque", Proceedings of ALNE $13 /$ NELS 13, 1983.

Levin, Beth C., On the Nature of Ergativity, dissertation de Ph. D., MIT, Cambridge, 1983.

Levin, Beth C., "The Basque verbal invezntory and configurationality", dans Muysken (édit.) Configurationality, Dordrecht, 1983.

Levin, Beth C. et Rappaport Hovav, Malka, Unaccusivity, Cambridge Mass., 1995.

Michelena, Luis, Diccionario General Vasco I..., Bilbao, 1987.

Oihartzabal, Beñat, "Structural Case and Inherent Case Marking: Ergaccusativity in Basque", dans Lakarra J. et Ortiz de Urbina, J., Syntactic Theory and Basque Syntax, 309-342, Saint-Sébastien, 1992.

Ortiz de Urbina, Jon, Parameters in the Grammar of Basque, Dordrecht, 1989.

Picoche, Jacqueline, Nouveau dictionnaire étymologique du français, Paris, 1971.

Rebuschi, Georges, "Autour du passif et de l'antipassif en basque biscayen", dans Paris, Catherine (édit.), Relations Prédicat-actant(s) dans des langues de types divers, II, 149-170, Paris, SELAF, 1979.

Rebuschi, Georges, Structure de l'énoncé en basque, Paris, SELAF, 1984.

Silverstein, Michael, "Chinook Jargon: Language Contact and the Problem of Multilevel Generative Systems, part I", Language, 48-2, 378-406, 1972.

Silverstein, Michael, "Hierarchy of Features and Ergativity", dans Dixon R.M.W. (édit.), Grammatical Categories in Australian Languages, 112-171, Canberra, 1976. 